\title{
The spatial transformation of color in the primary visual cortex of the macaque monkey
}

Elizabeth N. Johnson, Michael J. Hawken and Robert Shapley

Nat. Neurosci. 4, 409-416 (2001)

Part of a sentence in the legend to Fig. 7 was omitted. The correct figure legend appears below. We regret the error.
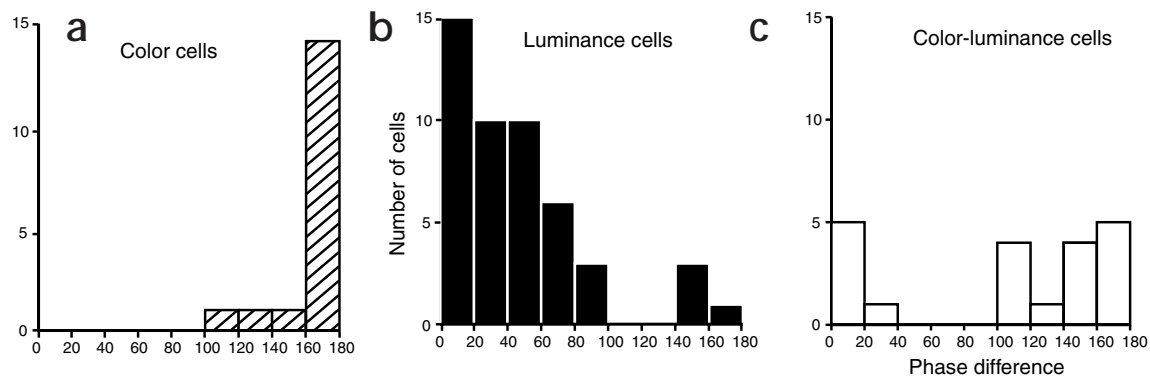

Fig. 7. $H$ istograms of the $L-$ and $M$-cone phase differences from the $L$ - and $M$-cone isolating frequency responses for all simple cells in the population. (a) The phase differences for neurons responding predominantly to equiluminance (color cells). (b) The phase differences for neurons responding predominantly to luminance (luminance cells). (c) The phase difference for neurons responding comparably to both chromatic equiluminance and luminance (color-luminance cells).

\section{Synapsin dispersion and reclustering during synaptic activity}

Ping Chi, Paul Greengard and Timothy A. Ryan

Nat. Neurosci. 4, 1187-1193 (2001)

Figures 1 and 2a and b reproduced poorly. The correct figures appear below. We regret the error.

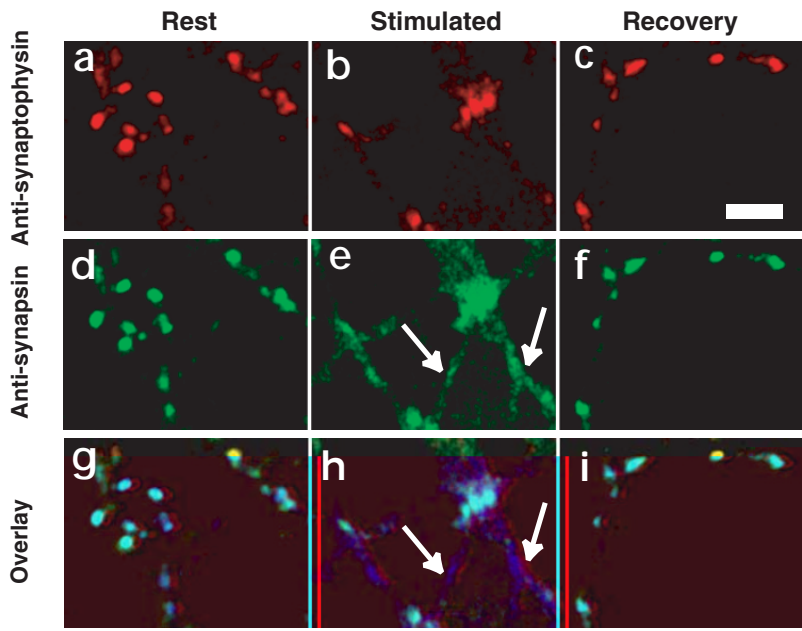

Fig. 1. Synapsin disperses from synaptic vesicles during activity. (a, d, $\mathbf{g})$ C 0 -immunolocalization of synaptophysin (red; a) and synapsin (green; $\mathbf{d}$ ) in hippocampal cell cultures fixed at rest show punctate staining for both markers (g). ( $\mathbf{b}, \mathbf{e}, \mathbf{h})$ In a parallel culture, fixed immediately after a train of a 900-AP stimulation at $10 \mathrm{~Hz}$, synapsin staining $(\mathbf{e}, \mathbf{h})$ is much more diffuse than synapto physin staining $(\mathbf{b}, \mathbf{h})$ indicating that synapsins dissociate from synaptic vesicles and redistribute into axons (arrows) in response to electrical stimulation. (c, f, i) In a different specimen fixed 10 min after stimulation, immunostaining of synapsin returns to a punctate pattern colocalized with synapto physin $(\mathbf{c}$, i), indicating a dynamic relocalization of synapsins from axons to synapses post-stimulation (f, i). Scale bar, $5 \mathrm{~mm}$.

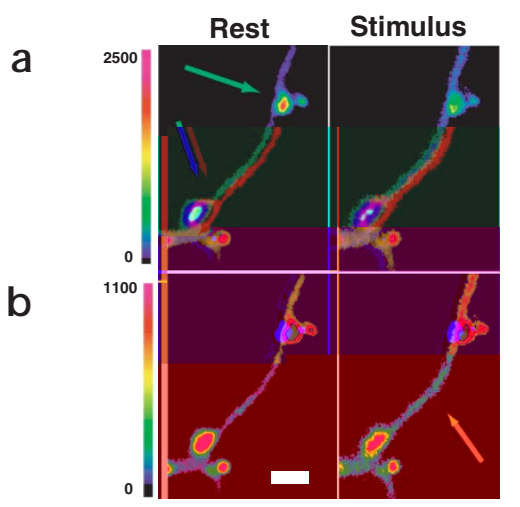

Fig. 2. Kinetics of GFP-synapsin la dispersion and recovery. (a) GFPsynapsin la fluorescence at synapses (green arrows) decreases upon stimulation. (b) GFP-synapsin la fluorescence in axons increases upon stimulation (red arrow). Same image as in (a) with different color scale to emphasize the fluorescence in the axon. The color scales show fluorescence intensity in arbitrary fluorescence units. Scale bar, $2 \mathrm{~mm}$. 